\title{
Differentiation of human round spermatids into motile spermatozoa through in vitro coculture with Vero cells
}

\author{
Atsushi Tanaka - Motoi Nagayoshi · \\ Shoichiro Awata · Izumi Tanaka · Hiroshi Kusunoki
}

Received: 6 April 2009/Accepted: 21 July 2009/Published online: 21 August 2009

(C) Japan Society for Reproductive Medicine 2009

\begin{abstract}
Purpose This study was undertaken to examine whether human early round spermatids will differentiate in an in vitro coculture with Vero cells.

Methods A total of 1450 and 400 isolated early round spermatids mechanically collected from two non-obstructive and three obstructive azoospermic men with a normal karyotype were cocultured on Vero cell monolayers in minimum essential medium plus $10 \%$ fetal bovine serum, with or without 50 or $100 \mathrm{IU} / \mathrm{L}$ FSH and 1 or $10 \mu \mathrm{mol} / \mathrm{L}$ testosterone, at $32.5^{\circ} \mathrm{C}$, in an environment of $5 \% \mathrm{CO}_{2}$ in air. Morphological changes of the spermatids were observed microscopically.

Results After 7 days of coculture, almost half (40-50\%) of the round spermatids from both non-obstructive and obstructive azoospermic men resumed spermiogenesis in vitro. Only cells from the latter patients gave rise to spermatozoa, a few of which had a motile flagellum. Low concentrations of FSH and testosterone increased the percentage of in vitro spermiogenesis.

Conclusions Isolated round spermatids can resume spermiogenesis in vitro when cocultured on a Vero cell monolayer.
\end{abstract}

A. Tanaka $(\bowtie) \cdot$ M. Nagayoshi $\cdot$ S. Awata $\cdot$ I. Tanaka

Saint Mother Obstetrics and Gynecology Clinic

and Institute for ART, 4-9-12 Orio, Yahata-Nishi,

Kitakyushu, Fukuoka 807-0825, Japan

e-mail: incho@stmother.com

H. Kusunoki

Faunal Diversity Sciences, Graduate School of Agriculture,

Kobe University, Rokkodai 1-1, Nada-ku, Kobe 657-8501, Japan
Keywords Coculture - Human - Spermiogenesis · Spermatids $\cdot$ Vero cells

\section{Introduction}

For patients with non-obstructive azoospermia, the spermiogenesis of which is completely arrested, the microinjection of round spermatids or their nuclei into oocytes (ROSI or ROSNI) is the only method that leads to fertilization. The success rate for this method, however, has been very low. It is thought that one of the reasons for this low rate is that round spermatids are too immature to activate the oocytes. There have been a few attempts to overcome this problem by the in vitro maturation of round spermatids [1-10], some of which have led to pregnancies and birth [10], but the clinical efficiency of this method is still poor. In such attempts, Cremades et al. [7] showed for the first time that co-culture on Vero cell monolayers could support full maturation of human round spermatids.

In this study, we verified and modified the coculture method reported by Cremades et al. [7], and succeeded in differentiating early round spermatids into normal late elongated spermatids with motile flagella.

\section{Materials and methods}

Ethical aspects

This study was conducted with the informed consent of all participating patients. The experimental use of human spermatogenic cells was approved by the Institutional Review Board of the Saint Mother Obstetrics and Gynecology Clinic. 
Patients and preparation of early round spermatids

Four $5-\mathrm{mm}^{3}$ pieces of testicular tissues were obtained through open biopsies from two non-obstructive and three obstructive azoospermic men, aged between 25 and 35 years. The results of preliminary biopsies indicated that spermatogenesis had been arrested at the level of the round spermatid in all non-obstructive azoospermic men. On other hand, a small number of mature spermatozoa were found in all obstructive azoospermic men. Testicular size and consistency, hormonal profiles (serum FSH, LH and testosterone) and the results of lymphotic karyotyping of these five patients are summarized in Table 1.

After being washed with erythrocyte-lysing buffer [11], the testicular tissues were finely minced with ophthalmic knives and filtered using a $0.22-\mu \mathrm{m}$ micro-filter. A few spermatozoa were detected in the resulting cell suspension from the obstructive azoospermia samples, but they were morphologically abnormal.

Isolated spermatogenic cells and Vero cells were observed. Micrographs of these cells were taken with a phase contrast microscope (Olympus, BH-2, Tokyo, Japan), an inverted microscope equipped with Hoffman modulation contrast optics (Nikon, TE300, Tokyo, Japan) or a Normarski differential interference contrast system (Olympus, IX71, Tokyo, Japan) on a temperature-controlled stage $\left(32.5\right.$ or $\left.37^{\circ} \mathrm{C}\right)$.

Early round spermatids were distinguished by the following cytological criteria: a cell diameter of about 6$8 \mu \mathrm{m}$, which is slightly larger than that of erythrocytes, and a nucleus/cytoplasm ratio of $80-90 \%$. In addition, round spermatids had a relatively thick nuclear membrane all around the nuclei and a fragile cytoplasmic membrane. This meant that when the spermatids were aspirated into a smaller size of microinjection pipette, their nuclei were easily detached intact from the cytoplasm in the pipette (Fig. 1a-c). In contrast, somatic cells such as lymphocytes, which are almost the same size as round spermatids, could not be easily aspirated into the pipette and, even if aspirated, they maintained their morphology in the pipette, even after been discharged (Fig. 1f-h). Moreover, if a spermatid was not viable, its nucleus was fragmented into pieces in the pipette (Fig. 1d, e), which was useful because it was necessary to select only viable spermatids for coculture. Furthermore, the presence of flattened large acrosomal vesicles was a definitive marker for cap and acrosomal phase spermatids (Fig. 2).

Preparation of Vero cell monolayers

Vero cell monolayers were prepared according to a previously described method [12]. In brief, the Vero cells used in our study were of the Vero Cell Line (WRC 0002 RIKEN, Japan). Frozen Vero cells were thawed, minimum essential medium (MEM) plus 10\% fetal bovine serum (FBS) was added, and the cells were then centrifuged at $800 \times g$ for 3 min. The supernatant was discarded and the cells were dispensed into culture bottles. The following day, the monolayer of Vero cells was treated with trypsin, and its concentration was adjusted to $10^{5}$ cells $/ \mathrm{mL}$. Microdrops of the Vero cell suspension were formed in $60-\mathrm{mm}$ culture dishes, which were then covered with mineral oil and cultured at $37^{\circ} \mathrm{C}$ in a gas phase of $\mathrm{O}_{2}: \mathrm{CO}_{2}: \mathrm{N}_{2}=5: 5: 90$. A few days later, the cells reached confluence. The initial period of co-culture is very important because immediately after the confluence, when the Vero cells monolayers are too active, they monopolize nutriments and some other components in the culture medium. So this period is not suitable for the survival and differentiation of round spermatids. In order to solve this issue, in this study we decided to start the coculture three to four weeks after the confluence when the Vero cells were in equilibrium state.

\section{Coculture of early round spermatids and Vero cells}

To facilitate observation of the morphological changes in the cultured early round spermatids, small square-shaped cuts were made in the underside of the bottom of the coculture dishes. By using micromanipulators (Narishige, Japan), 50 early round spermatids were separately placed on confluent

Table 1 Patient characteristics

\begin{tabular}{|c|c|c|c|c|c|c|c|c|}
\hline \multirow[t]{2}{*}{ Symptoms } & \multirow[t]{2}{*}{ Patient no. } & \multirow[t]{2}{*}{ Age (years) } & \multicolumn{2}{|l|}{ Testis } & \multicolumn{3}{|l|}{ Serum } & \multirow{2}{*}{$\begin{array}{l}\text { Lymphotic } \\
\text { karyotype }\end{array}$} \\
\hline & & & Size & Consistency & $\begin{array}{l}\text { FSH } \\
(\mathrm{mIU} / \mathrm{mL})\end{array}$ & $\begin{array}{l}\mathrm{LH} \\
(\mathrm{mIU} / \mathrm{mL})\end{array}$ & $\begin{array}{l}\mathrm{T} \\
(\mathrm{ng} / \mathrm{dL})\end{array}$ & \\
\hline \multirow[t]{2}{*}{ Non-obstructive azoospermia } & 1 & 31 & Small & Hard & 25.2 & 5.0 & 421 & Normal $^{\mathrm{a}}$ \\
\hline & 2 & 34 & Slightly small & Normal & 12.1 & 3.0 & 380 & Normal $^{\mathrm{a}}$ \\
\hline \multirow[t]{3}{*}{ Obstructive azoospermia } & 3 & 34 & Normal & Normal & 8.0 & 1.9 & 327 & Normal $^{\mathrm{a}}$ \\
\hline & 4 & 35 & Normal & Normal & 3.0 & 1.7 & 305 & Normal $^{\mathrm{a}}$ \\
\hline & 5 & 25 & Normal & Normal & 5.6 & 3.4 & 363 & Normal $^{\mathrm{a}}$ \\
\hline
\end{tabular}

a $46 X Y$ 


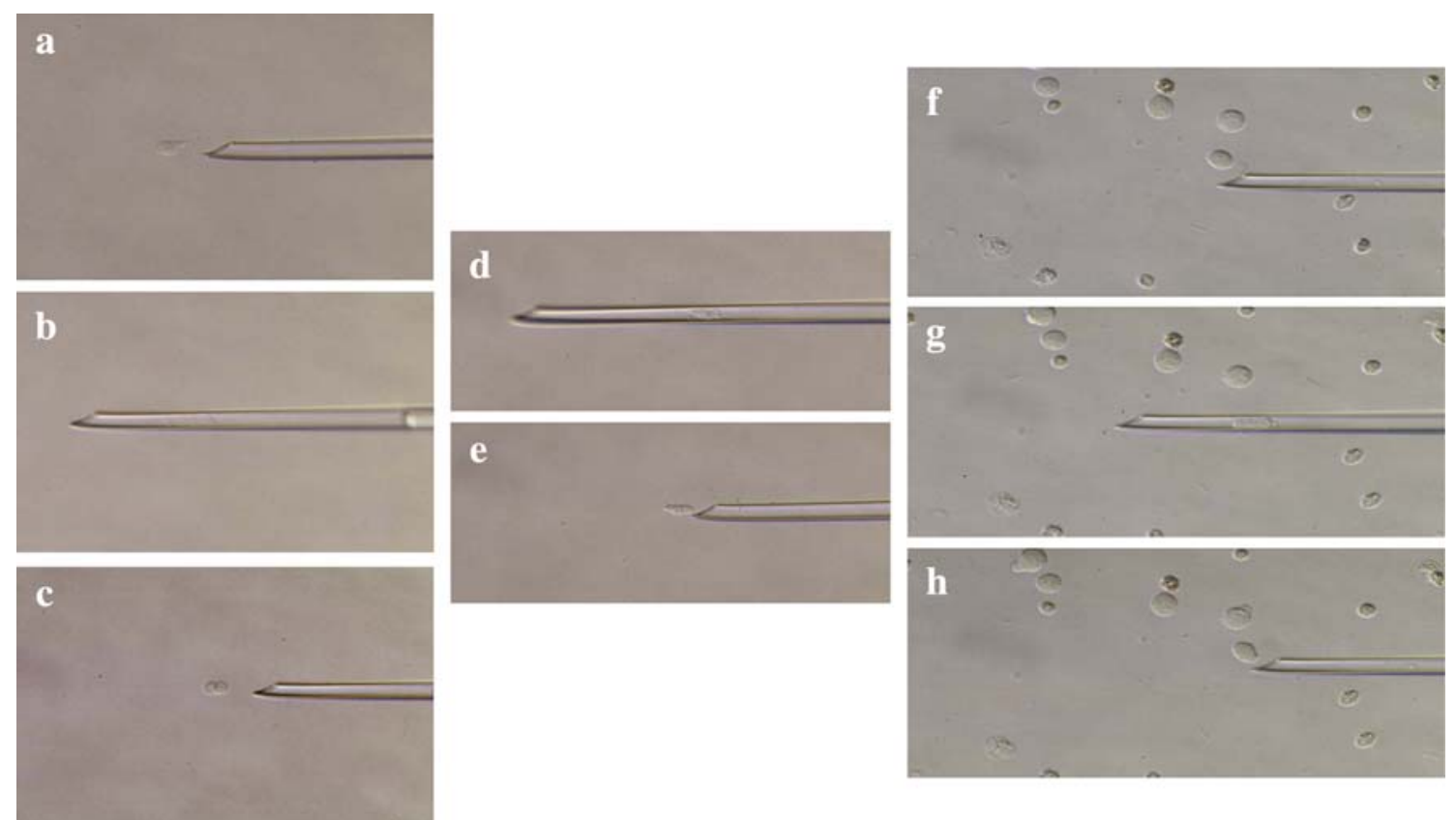

Fig. 1 Selecting viable round spermatids $(\mathbf{a}-\mathbf{c})$ from dead ones $(\mathbf{d}, \mathbf{e})$ and somatic cells $(\mathbf{f}-\mathbf{h})$ using the aspiration method, original magnification, $\times 200$

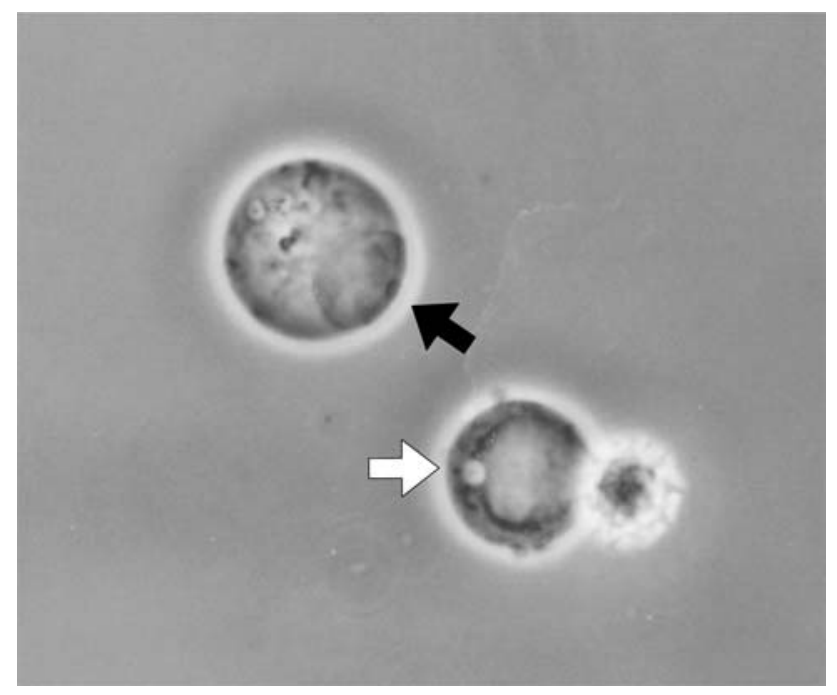

Fig. 2 Isolated early round spermatids with acrosomal granules (black arrow) and vesicles (white arrow), original magnification, $\times 1,000$

feeder layers in these areas (Figs. 3, 4) and cocultured with MEM plus $10 \%$ FBS, with or without 50 or $100 \mathrm{IU} / \mathrm{L}$ FSH and 1 or $10 \mu \mathrm{mol} / \mathrm{L}$ testosterone, for 7 days at $32.5^{\circ} \mathrm{C}$, in an environment of $5 \% \mathrm{CO}_{2}$ in air. Half of the culture medium was replaced every $48 \mathrm{~h}$, and observations were made twice daily (morning and evening). During coculture, the spermatids remained in the same position on the monolayer, because they were loosely attached to the Vero cells.

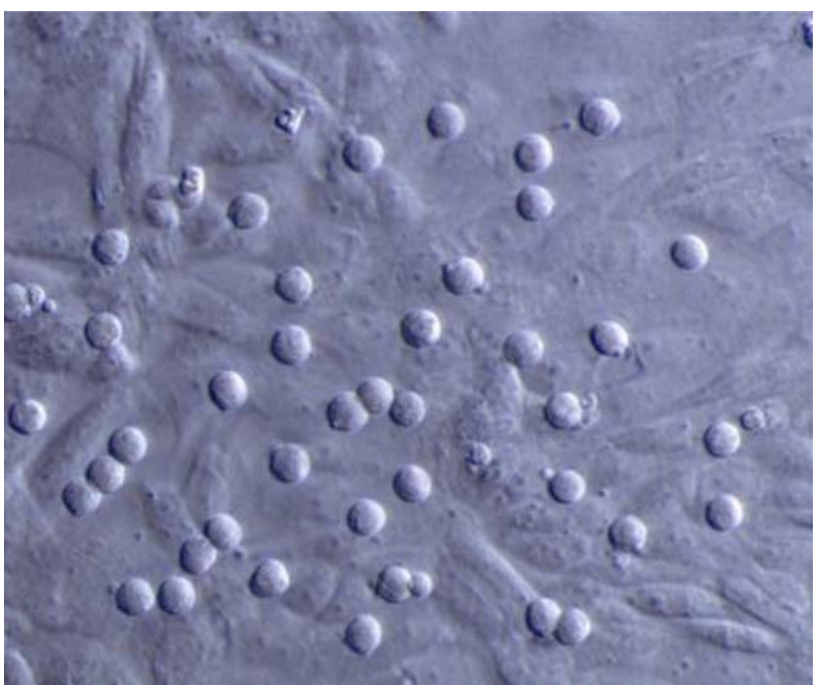

Fig. 3 Early round spermatids on the monolayer of Vero cells, original magnification, $\times 400$

Vero cells were easily discernible from early round spermatids, because they were about $10 \mu \mathrm{m}$ in diameter, much larger than the round spermatids. In addition, the surface and cytoplasm of the Vero cells were rough and thick, respectively, and many intracellular granules could be seen inside them. Furthermore, the Vero cells adhered to the feeder layer so tightly that it was difficult to detach them from the layer via aspiration. 
Fig. 4 Schematic illustration of the coculture system

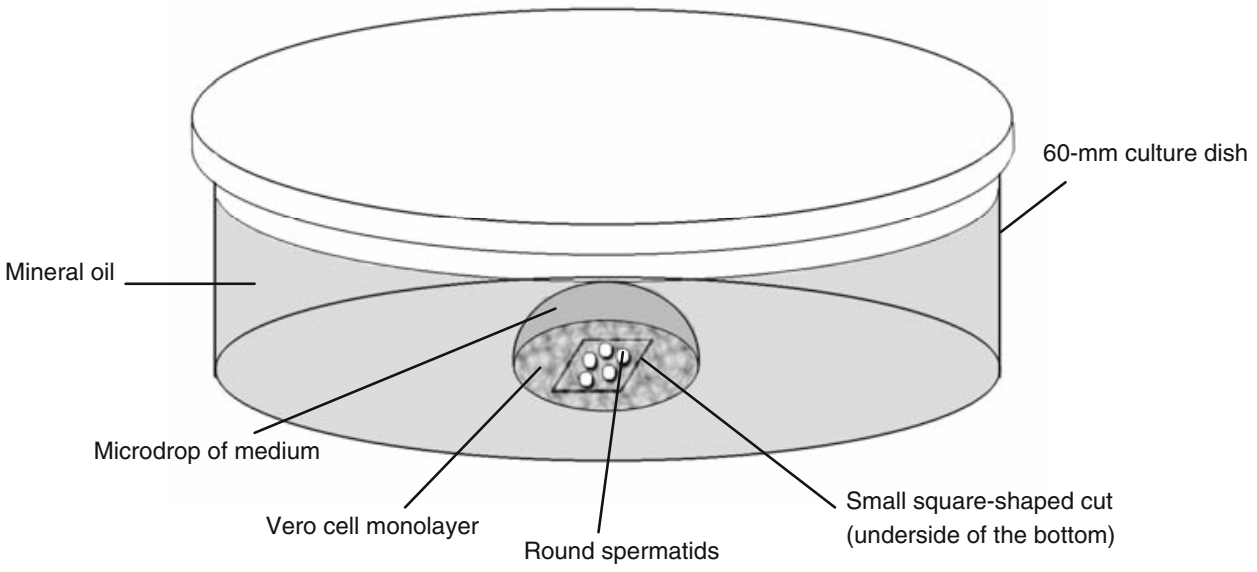

Statistical analysis

The $\chi^{2}$-test was used to evaluate whether differences in the percentages of spermatids or spermatozoa with various morphologies was significant.

\section{Results}

Changes in the early round spermatids obtained from the two non-obstructive and three obstructive azoospermic men during in vitro spermiogenesis under various coculture conditions are summarized in Table 2. During in vitro spermiogenesis, the same changes that occur during in vivo spermiogenesis took place: namely, nucleus condensation and protrusion, flagellar growth and movement (Fig. 5).

For the non-obstructive azoospermia samples, out of 100 early round spermatids cocultured for 7 days with Vero cells in MEM plus 10\% FBS without hormones, 41 cells (41.0\%) differentiated into elongating spermatids (Fig. 5a). When the culture medium was supplemented with low concentrations of FSH (50 IU/L) and testosterone $(1 \mu \mathrm{mol} /$ $\mathrm{L})$, the differentiation rate rose to $50.0 \%(75 / 150)$. However, higher concentrations of these hormones (100 IU/L FSH and/or $10 \mu \mathrm{mol} / \mathrm{L}$ testosterone) led to lower differentiation rates (22.0-36.0\%). No spermatids differentiated into an elongated stage under any of the culture conditions used.

For the obstructive azoospermia samples, out of 350 early round spermatids cocultured for 7 days in medium without hormones, 146 cells $(41.6 \%)$ differentiated into elongating spermatids, $3(0.9 \%)$ into elongated spermatids (Fig. 5b) and 17 (4.9\%) into spermatozoa (Fig. 5c, d). When low concentrations of hormones were added to the medium, the rate of differentiation into elongating and elongated spermatids, and spermatozoa rose to $48.6 \%$ (462/ 950), $12.8 \%$ (122/950) and 5.3\% (50/950), respectively.
Within these groups, only a few spermatozoa $(0.2-0.6 \%)$ had a motile flagellum (Fig. 5d). When higher concentrations of hormones were used, the differentiation rates were lower or almost the same (40.0-48.0, 6-16 and 4-8\%, respectively), but none of the cells had a motile flagellum.

\section{Discussion}

In our clinic, the normal fertilization rate of 58.9\% (106/ $180)$ and cleavage rate of $40.6 \%$ (73/180) after ROSI, using cells obtained from patients whose most advanced germ cells in their seminiferous tubules are a few late spermatids or spermatozoa, are almost equal to or higher than those rates using cells from patients whose most advanced germ cells are round spermatids $(61.8 \%, 157 / 254$; and $30.7 \%$, 78/254; respectively) (unpublished). It has been reported that there is a marked difference in the clinical outcomes of ROSI using late spermatids and ROSI using early spermatids [13]. Taking these findings into account, two procedures can be employed in order to improve the success rate of ROSI and ROSNI: first, the oocytes can be activated by electric stimulation [14] prior to ROSI or ROSNI; and second, an immature spermatid can be grown to maturity in vitro before being used in ROSI or ROSNI.

Cremades et al. [7] reported success in growing early round spermatids into mature late elongated spermatids when the cells were obtained from patients with elongating or elongated spermatids in their seminiferous epithelium and cocultured on a Vero cell monolayer. However, these late elongated spermatids were thought to fall into the $\mathrm{Sd} 2$ category of Clermont [15] and were immotile (personal communication). We have also cocultured early round spermatids collected from a young mouse (14 days old) on a monolayer of Sertoli cells collected from another young mouse; however, spermiogenesis did not resume (unpublished). In this study, Vero cells, which are derived from 


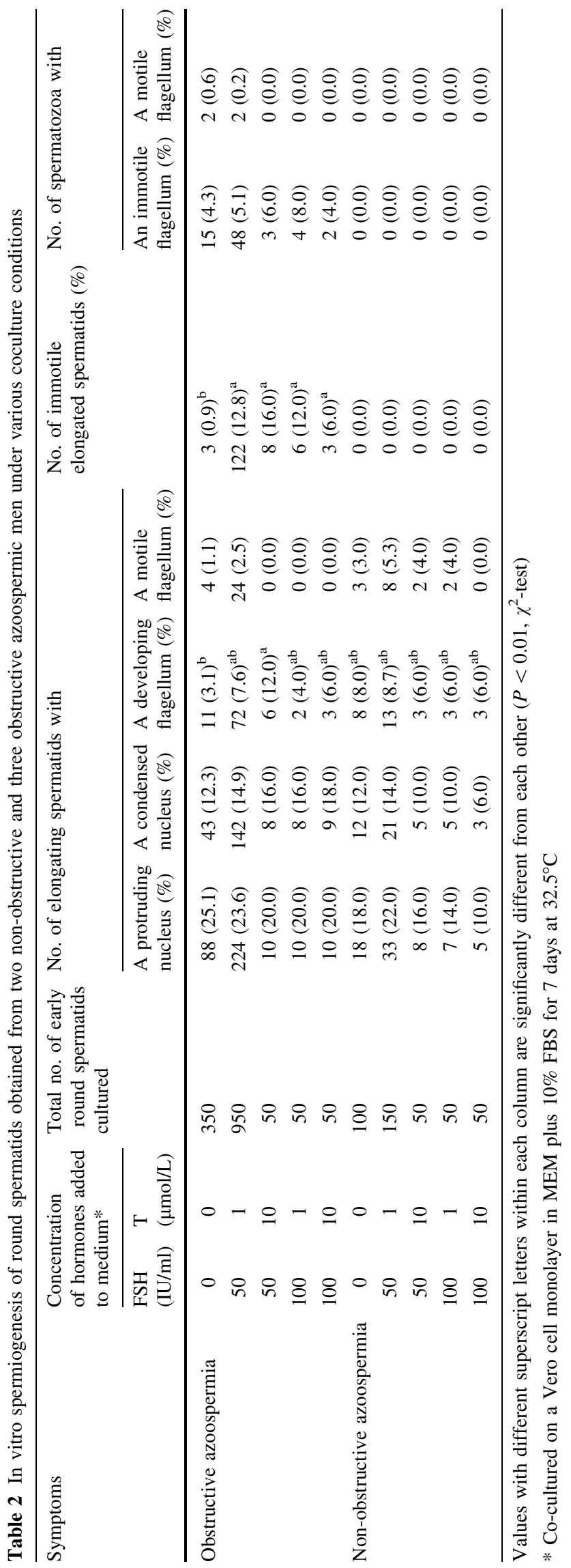

the kidney of African green monkeys, were used as feeder cells instead of Sertoli cells, resulting in successful in vitro spermiogenesis. Although the reason for this success is uncertain, because the kidney is equivalent to the testis in terms of its mesoderm origin, it may be related to the effectiveness of Vero cells.

We improved the temperature and the starting time of the coculture described by Cremades et al. [7]. In their report, culturing was carried out at $37^{\circ} \mathrm{C}$ and Vero cells were used for coculturing as soon as they became confluent. Here, we first reduced the culture temperature to $32.5^{\circ} \mathrm{C}$, given that the testicular temperature is lower than the internal body temperature. The importance of the culture temperature for successful in vitro spermatogenesis has been previously described by Tesarik et al. [10] and Cremades et al. [8]. Second, instead of using the Vero cells soon after they reached confluence, we left them for a further 3-4 weeks. We think that proliferating Vero cells may consume nutrients that are necessary for spermatids and may therefore interfere with their development. Furthermore, we suspect that the most effective factors needed for in vitro spermiogenesis, for example growth factors, are secreted from Vero cells after 3-4 weeks rather than immediately after confluence. By making these improvements, we succeeded in achieving more spermatids that could resume spermiogenesis.

We must consider the safety of Vero cells. Controlling infections is critically important when applying heterozoically derived cells to clinical use. Cremades et al. [7] emphasized that Vero cells are useful for supporting the full maturation of human round spermatids in vitro but they present a drawback for clinical application because of the inherent risk of infection. The World Health Organization (WHO) urges that due attention should be paid in checking for infections when using new Vero cells and recommends that established cell lines that have been in use for a long time should be used rather than new cell lines. The WHO also recommends refraining from the use of cells that have had 162 or more inoculations, because such cells take on a tumorigenic nature. Conventionally, coculture with Vero cells has been used to improve the development of human embryos [16] and the maturation of human follicular oocytes in vitro [17]. On the basis of these findings, we consider that there is very little risk of infection associated with the use of Vero cells. In any case, the full informed consent of patients who are ready to receive assisted reproduction treatment using immature spermatogenic cells is very important.

We examined two groups of patients in this study: the first was non-obstructive azoospermic men, whose spermatogenesis was arrested at the round spermatid level; and the second was obstructive azoospermic men, in the testicular cell suspension of whom a few immotile 
Fig. 5 Elongating and elongated spermatids (a-d) resulting from the in vitro differentiation of isolated early round spermatids, original magnification, $\times 600$ (a) and $\times 400$ (b-d). a Early elongating spermatids showing nucleus protrusion (black arrows), nucleus condensation (arrowheads) and flagellar growth (white arrow), b a late elongated spermatid with an immotile flagellum (arrow), c a spermatozoon with an immotile flagellum (arrow), and $\mathbf{d}$ a spermatozoon with a motile flagellum (arrow)
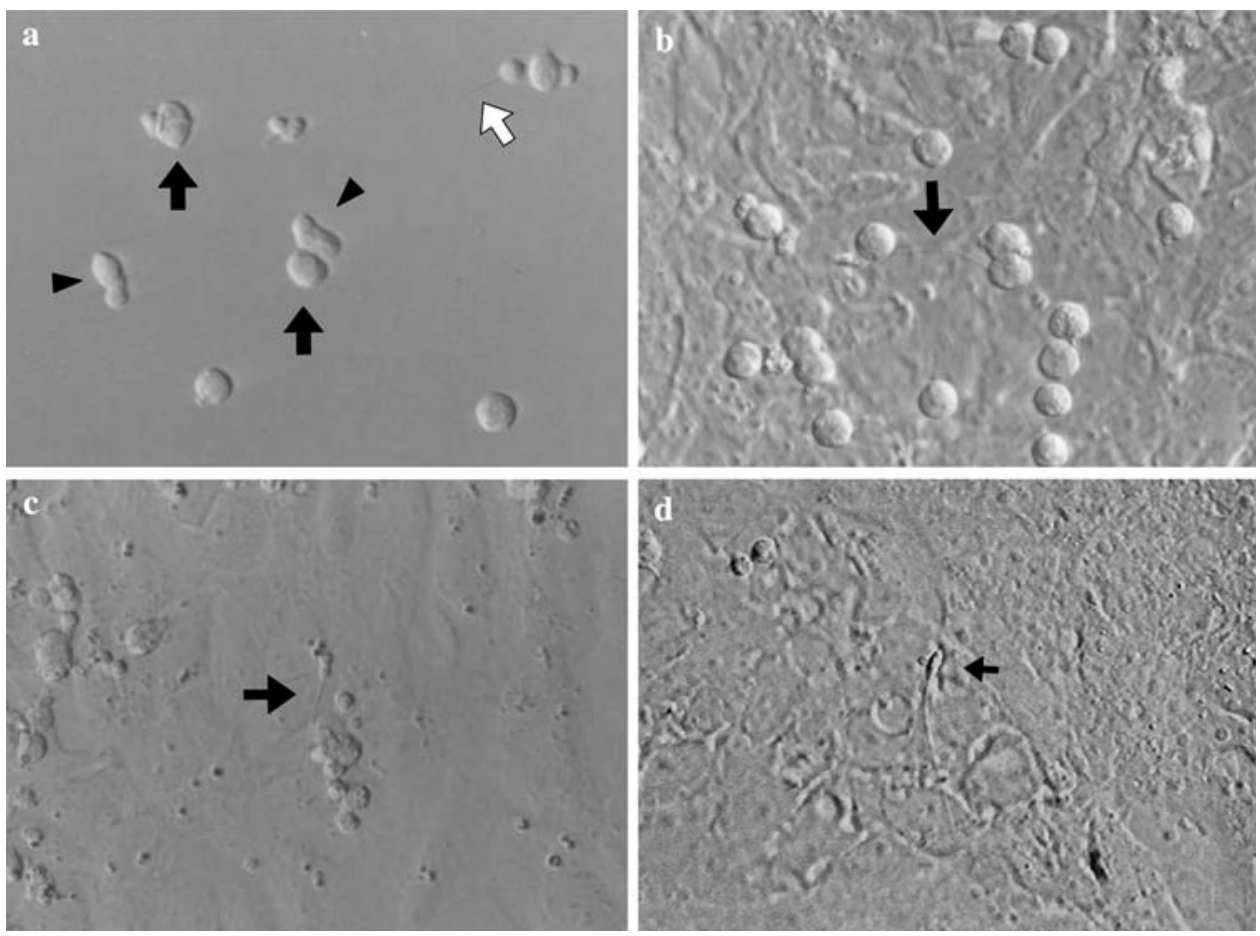

spermatozoa were detected. The coculture results were different for these two groups. In the second group, approximately $40-50 \%$ of the early round spermatids resumed spermiogenesis in vitro and resulted in normal spermatozoa with a motile flagellum. By contrast, in the first group, which had almost an equal proportion of spermatids resuming spermiogenesis, we could detect no late elongated spermatids or spermatozoa with or without a motile flagellum. Cremades et al. [8] reported a similar tendency. They stated that early round spermatids from obstructive azoospermic patients that were cultured in Vero cell-conditioned medium had relatively higher maturation rates than those from non-obstructive azoospermic patients. It is thought that a defect in the spermatid differentiating capacity leading to maturation arrest is the cause of these differences. For such patients, we will have to research further the culture method.

In this study, in vitro spermiogenesis was improved by including a low concentration of FSH and testosterone in the culture medium. Tesarik et al. [9] reported that isolated primary and secondary spermatocytes and early round spermatids from patients with very high serum FSH concentrations $(>20 \mathrm{IU} / \mathrm{L})$ resumed spermatogenesis in vitro to a lesser extent than those from men with low serum FSH concentrations (10-20 IU/L), when clusters of such germ cells, including Sertoli cells, were cultured for $24 \mathrm{~h}$. They also reported that adding recombinant $\mathrm{FSH}$ to the culture medium promoted the in vitro differentiation of germ cells obtained from patients with obstructive azoospermia [6]. Testosterone, although it was ineffective alone, improved the effectiveness of FSH on in vitro meiosis and spermiogenesis [5]. In the presence of high concentrations of FSH and testosterone, however, human spermatogenesis proceeded in vitro at an unusual speed, and the resulting germ cells were morphologically abnormal [5]. Tesarik et al. [5] suggested that the effects of testosterone were probably due to the prevention of Sertoli cell apoptosis. In our coculture system, the effects of testosterone on Vero cells have not yet been determined.

\section{References}

1. Barros A, Bernabeu R, Takahashi K, et al. Intracytoplasmic injection of ejaculate and testicle spermatids: report on 35 cycles. Hum Reprod. 1998;13(Abstract book 1):154-5.

2. Barros A, Takahashi K, Bernabeu R, et al. Spermatid intracytoplasmic injection: report on 56 cycles. Fertil Steril. 1998; (Abstract Suppl.): S441.

3. Bernabeu R, Cremades N, Takahashi K, Sousa M. Successful pregnancy after spermatid injection. Hum Reprod. 1998;13: 1898-900.

4. Aslam I, Fishel S. Short-term in-vitro culture and cryopreservation of spermatogenetic cells used for human in-vitro conception. Hum Reprod. 1998;13:634-8.

5. Tesarik J, Guido M, Mendoza C, Gerco E. Human spermatogenesis in vitro: respective effects of follicle-stimulating hormone and testosterone on meiosis, spermiogenesis, and Sertoli cell apoptosis. J Clin Endocrinol Metab. 1998;33:4467-73.

6. Tesarik J, Gerco E, Rienzi L, et al. Differentiation of spermatogenic cells during in-vitro culture of testicular biopsy samples from patients with obstructive azoospermia: effect of recombinant follicle stimulating hormone. Hum Reprod. 1998;13:2772-81. 
7. Cremades N, Bernabeu R, Barros A, Sousa M. In-vitro maturation of round spermatids using co-culture on Vero cells. Hum Reprod. 1999;14:1287-93.

8. Cremades N, Sousa M, Bernabeu R, Barros A. Developmental potential of elongating and elongated spermatids obtained after in-vitro maturation of isolated round spermatids. Hum Reprod. 2001;16:1938-44.

9. Tesarik J, Balaban B, Isikal A, et al. In-vitro spermatogenesis resumption in men with maturation arrest: relationship with in-vivo blocking stage and serum FSH. Hum Reprod. 2000;15:1350-4.

10. Tesarik J, Bahceci M, Ozcan C, Greco E, Mendoza C. Restoration of fertility by in-vitro spermatogenesis. Lancet. 1999; 353:555.

11. Verheyen G, De Croo I, Tournaye H, Pletincx I, Devroey P, van Steirteghem AC. Comparison of four mechanical methods to retrieve spermatozoa from testicular tissue. Hum Reprod. 1995;10:2956-9.

12. Tanaka A, Nagayoshi M, Awata S, Mawatari Y, Tanaka I, Kusunoki $H$. Completion of meiosis in human primary spermatocytes through in vitro coculture with Vero cells. Fertil Steril. 2003;79(Suppl 1):795-801.

13. Vanderzwalmen $\mathrm{P}, \mathrm{Nijs} \mathrm{M}$, Stecher A, et al. Is there a future for spermatid injections? Hum Reprod. 1998;13(Suppl 4):71-84.

14. Sofikitis NV, Toda T, Miyagawa I, Zavos PM, Pasyianos P, Mastelou E. Beneficial effects of electrical stimulation before round spermatid nuclei injections into rabbit oocytes on fertilization and subsequent embryonic development. Fertil Steril. 1996;65:176-85.

15. Clermont Y. The cycle of the seminiferous epithelium in man. Am J Anat. 1963;112:35-51.

16. Menezo YJ, Guerin JF, Czyba JC. Improvement of human embryo development in vitro by coculture on monolayers of Vero cells. Biol Reprod. 1990;42:301-6.

17. Cha KY, Han SY, Chung HM, et al. Pregnancies and deliveries after in vitro maturation culture followed by in vitro fertilization and embryo transfer without stimulation in women with polycystic ovary syndrome. Fertil Steril. 2000;73:978-83. 